\title{
The LTP interferometer and phasemeter
}

\author{
G Heinzel $^{1}$, V Wand ${ }^{1}$, A García ${ }^{1}$, O Jennrich ${ }^{2}$, C Braxmaier ${ }^{3}$, \\ D Robertson ${ }^{4}$, K Middleton ${ }^{5}$ D Hoyland ${ }^{6}$, A Rüdiger ${ }^{1}$, R Schilling ${ }^{1}$, \\ U Johann ${ }^{3}$ and K Danzmann ${ }^{1,7}$ \\ ${ }^{1}$ Max-Planck-Institut für Gravitationsphysik (Albert-Einstein-Institut), Callinstrasse 38, \\ D-30167 Hannover, Germany \\ 2 ESTEC, Noordwijk, The Netherlands \\ 3 Astrium GmbH, 88039 Friedrichshafen, Germany \\ ${ }^{4}$ Department of Physics and Astronomy, University of Glasgow, Glasgow, UK \\ ${ }^{5}$ Rutherford Appleton Laboratories, Chilton, UK \\ ${ }^{6}$ University of Birmingham, Birmingham, UK \\ ${ }^{7}$ Universität Hannover, Institut für Atom- und Molekülphysik, Callinstrasse 38, \\ D-30167 Hannover, Germany \\ E-mail: gerhard.heinzel@aei.mpg.de
}

Received 19 September 2003

Published 6 February 2004

Online at stacks.iop.org/CQG/21/S581 (DOI: 10.1088/0264-9381/21/5/029)

\begin{abstract}
The LISA Technology Package (LTP), to be launched by ESA in 2006/2007, is a technology demonstration mission in preparation for the LISA space-borne gravitational wave detector. A central part of the LTP is the optical metrology package (heterodyne interferometer with phasemeter) which monitors the distance between two test masses with a noise level of $10 \mathrm{pm} \mathrm{Hz}^{-1 / 2}$ between $3 \mathrm{mHz}$ and $30 \mathrm{mHz}$. It has a dynamic range of $>100 \mu \mathrm{m}$ without any actuators for the pathlength. In addition to the longitudinal measurements, it provides alignment measurements with an expected noise level of $<10 \operatorname{nrad~} \mathrm{Hz}^{-1 / 2}$. While the basic design has been described previously by Heinzel et al (2003 Class. Quantum Grav. 20 S153-61), this paper gives new details on the laser stabilization, the phasemeter and recent prototype results.
\end{abstract}

PACS numbers: $07.60 . \mathrm{Ly}, 07.87 .+\mathrm{v}$, 43.30.Rx, 04.80.Nn, 06.30.Bp, 06.30.Gv

\section{Introduction}

The LISA Pathfinder spacecraft will contain a European LISA Technology Package (LTP) and a similar US-supplied package (ST-7). Both packages consist of two free-floating test masses, each in capacitive sensor cages ('gravitational reference sensors'), which are the heart of the drag-free control and an essential part of the LISA mission [1]. The European package LTP and the US package ST-7 [2] will have coordinated differences in construction so as to maximize the scientific returns of the mission. The purpose of both packages is to test a variety of operational modes of the gravitational reference sensors together with their associated 


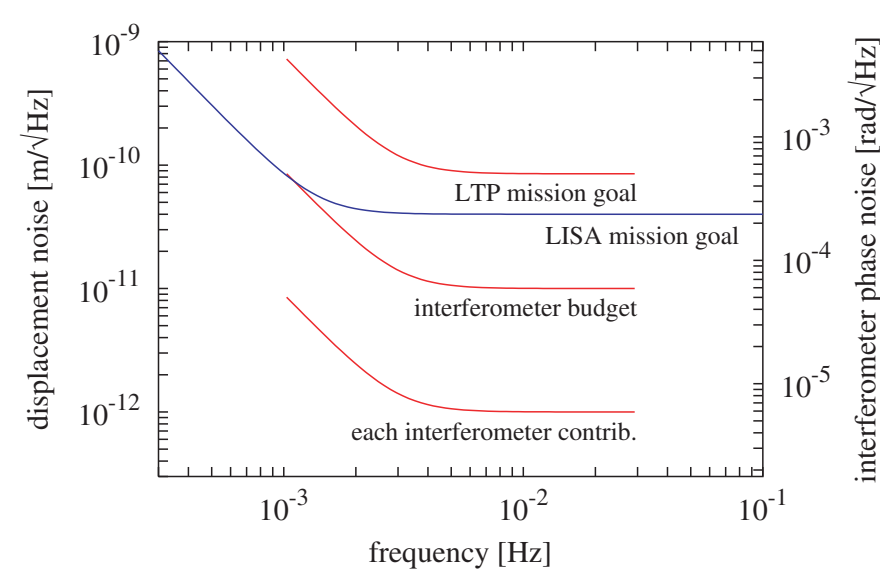

Figure 1. Noise budgets for the LTP, its interferometer and each individual interferometer noise source. The LISA total noise budget is also shown for comparison.

$\mu \mathrm{N}$-thrusters and drag-free loops, and to verify their performance and noise behaviour. Both packages will be launched together in 2006/2007 on an ESA satellite, the LISA Pathfinder mission (formerly called SMART-2).

The interferometer of the LTP is the diagnostic tool that is used to continuously monitor the test masses in all operating modes by measuring

- the distance between the two test masses (called $x_{1}-x_{2}$ ),

- the position of one test mass with respect to the optical bench (called $x_{1}$ ),

- the differential alignment of the two test masses (with two sets of measurements: DC and differential wavefront sensing (DWS)),

- the alignment of one test mass with respect to the optical bench.

Its principle of operation and general design are described in [3]. This paper describes in more detail the laser power stabilization, laser frequency noise control, the method of measuring the phase between heterodyne beat notes ('phasemeter') and the results of a laboratory prototype interferometer and phasemeter in Hannover.

\section{Laser power stabilization}

There are two separate requirements for laser power stability: radiation pressure noise on the test mass in the measurement band $(1-30 \mathrm{mHz})$ and direct coupling into the phase measurement at the heterodyne frequency (a few $\mathrm{kHz}$ ). For both of these the budget allocated to each individual interferometer noise source, namely $\tilde{\delta x}<1 \mathrm{pm} \mathrm{Hz}^{-1 / 2}$ or equivalently $\widetilde{\delta \varphi}<2 \pi \times 10^{-6} \mathrm{rad} \mathrm{Hz}^{-1 / 2}$ at $3 \mathrm{mHz}$, is allowed (see figure 1).

The light power $P$ reflected from the test masses produces a force on the masses. Fluctuations $\delta P$ in the light power will thus produce fluctuating forces on the gravitational sensors which could limit the sensitivity of the measurement. The displacement fluctuation $\delta x$ is given by

$$
\delta x=\frac{2 \delta P}{m c \omega^{2}},
$$

where $m$ is the test mass (assumed to be $2 \mathrm{~kg}$ ) and $\omega$ the Fourier frequency of the fluctuation. Accordingly, the required relative power stability for $2 \mathrm{~mW}$ of light in the measurement 


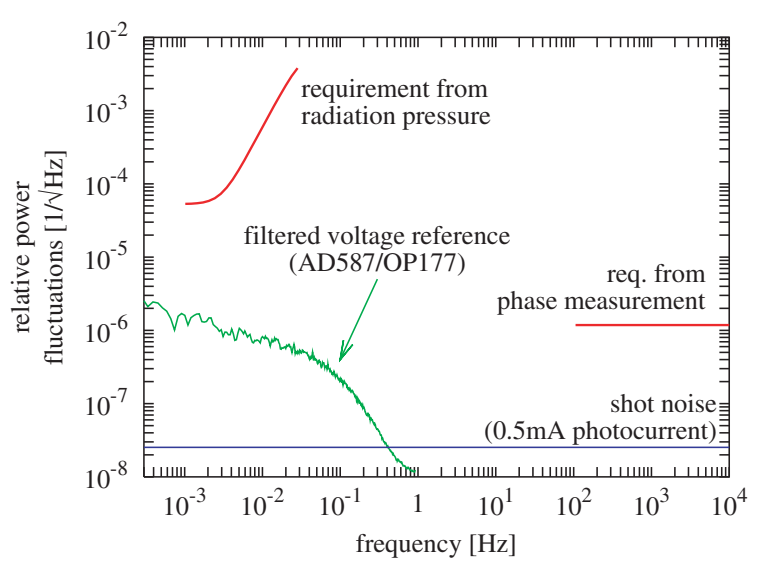

Figure 2. Laser power stability requirements. The upper two segments are the requirements, while the lower two lines are typical limits of the stabilization effort.

arm is

$$
\frac{\widetilde{\delta P}}{P}<\frac{m c \omega^{2}}{2 P} \tilde{\delta x} \approx 5 \times 10^{-5} \mathrm{~Hz}^{-1 / 2}
$$

between $1 \mathrm{mHz}$ and $3 \mathrm{mHz}$, relaxing as $f^{2}$ for frequencies up to $30 \mathrm{mHz}$ (see figure 2).

At the heterodyne frequency $f_{\text {het }}$, of some $\mathrm{kHz}$, the requirement is

$$
\frac{\widetilde{\delta P}}{P}<\frac{\eta}{2 \sqrt{2}} \widetilde{\delta \varphi} \approx 1.8 \times 10^{-6} \mathrm{~Hz}^{-1 / 2}
$$

for one phase measurement, with $\eta \lesssim 1$ the interferometric contrast, and it must be a factor of $\sqrt{2}$ smaller for the displacement measurements $x_{1}-x_{2}$ and $x_{1}$ that are obtained by subtracting two independent phase measurements.

In any case, stabilization of the laser power will be necessary. In order to compensate fluctuations of the fibre coupling efficiency and fibre transmission, it will be done by measuring the power at the end of each fibre and feeding a correction signal back to the respective AOM driver. Common mode signals may also be fed back directly to the laser pump.

Figure 2 shows these two requirements (radiation pressure and phase measurement). Also shown are two typical limits to the achievable stability: the noise of a voltage reference (an AD587 in unstabilized laboratory conditions with an additional OP177 lowpass filter) and the shot noise in a photocurrent of $0.5 \mathrm{~mA}$. Both of these limits are well below the required stability, so that the stabilization should present no major obstacle.

\section{Laser frequency fluctuations}

In the Mach-Zehnder interferometer, laser frequency fluctuations $\delta v_{\mathrm{L}}=\delta \omega_{\mathrm{L}} /(2 \pi)$ cause spurious phase fluctuations $\delta \varphi$ via a pathlength difference $\Delta l$ between the arms. The conversion factor from $\delta \omega_{\mathrm{L}}\left(\mathrm{rad} \mathrm{s}^{-1}\right)$ to $\delta \varphi(\mathrm{rad})$ is given by $\tau=\Delta l / c$, the differential time delay.

With the usual budget allocation of $\widetilde{\delta \varphi}<2 \pi \times 10^{-6} \mathrm{rad} \mathrm{Hz}^{-1 / 2}$ between $3 \mathrm{mHz}$ and $30 \mathrm{mHz}$, we obtain the frequency stability requirement

$$
\widetilde{\delta \nu_{\mathrm{L}}}<\frac{c}{2 \pi \Delta l} \widetilde{\delta \varphi}=28 \frac{\mathrm{kHz}}{\sqrt{\mathrm{Hz}}} /\left[\frac{\Delta l}{1 \mathrm{~cm}}\right],
$$




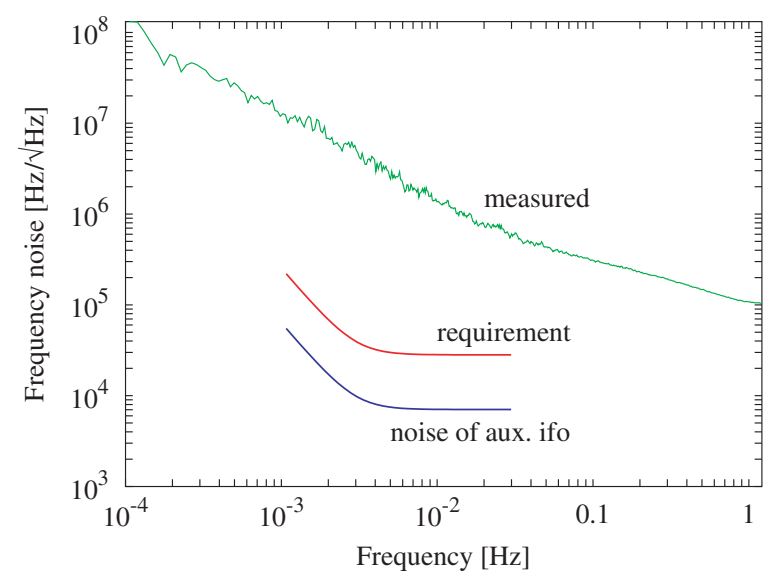

Figure 3. Measured free-running laser frequency noise and stability requirement. The lowest curve is the predicted noise of the auxiliary unequal-arm interferometer when used as a frequency fluctuation sensor.

which is shown together with the measured frequency noise of the unstabilized laser engineering model (EM) in figure 3. A suppression of the frequency fluctuations by a factor of about 100 is necessary.

Using a separate stabilization system, such as a cavity or an iodine cell, is too complex. Instead, an auxiliary interferometer on the optical bench [1] is used to sense the laser frequency fluctuations. It has an intentional pathlength difference of $\Delta L=40 \mathrm{~cm}$ and is otherwise similar to the main interferometers. Its predicted noise level is also shown in figure 3 . There are two options for using this frequency fluctuation signal.

- Using a feedback loop to actively stabilize the laser. The required loop gain is $\approx 100$ at $30 \mathrm{mHz}$. With a $1 / f$ simple integrator as loop filter we need a unity gain frequency $>3 \mathrm{~Hz}$. Allowing an extra phase delay of $45^{\circ}$ in the loop gain at $3 \mathrm{~Hz}$, the permissible processing time delay is $40 \mathrm{~ms}$, which is compatible with the phasemeter design. At DC the gain must be reduced again, to avoid forcing the laser to follow drifts of the auxiliary interferometer.

- Do not stabilize the laser but use the frequency fluctuation signal in a post-measurement correction of the main output signals. The actual pathlength differences $\Delta l$ must be known to relatively high precision $(\approx 0.1 \mathrm{~mm})$. Manufacturing to such accuracy is difficult, but measurement by calibration during operation is possible.

Both these options seem feasible, and the required hardware for both of them is identical (D/A converters in the phasemeter that connect to frequency control inputs of the laser head) and will be available, so that by appropriate software both can be used. Recently, it has been decided to use the active stabilization as baseline and the post-measurement correction as fallback option.

\section{Phasemeter}

The phasemeter must measure the phase between two electrical signals at $f_{\text {het }} \approx 1-2 \mathrm{kHz}$ with an output rate of $f_{\mathrm{ph}} \approx 100 \mathrm{~Hz}$. Out of several possible methods to measure the phase, we have chosen the single-bin discrete Fourier transform (SBDFT) method. Each signal 
is independently digitized by a 16 -bit $\mathrm{A} / \mathrm{D}$ converter at a sampling frequency $f_{\text {samp }} \approx 50$ $100 \mathrm{kHz}$. The resulting time series in a channel called A, $y_{i}^{A}$, is reduced to only three real numbers (at $\left.f_{\mathrm{ph}}\right)$ in a preprocessing stage:

DC components (real) : $\quad \mathrm{DC}^{A}, \ldots: \quad \mathrm{DC}^{A}=\sum_{i=0}^{n-1} y_{i}^{A}$,

$f_{\text {het }}$ components (complex) : $\quad \mathrm{F}^{A}, \ldots: \quad \operatorname{Re}\left(\mathrm{F}_{A}\right)=\sum_{i=0}^{n-1} y_{i}^{A} c_{i}, \quad \operatorname{Im}\left(\mathrm{F}_{A}\right)=\sum_{i=0}^{n-1} y_{i}^{A} s_{i}$.

The constants $s_{i}$ and $c_{i}$ are pre-computed as $c_{i}=\cos \left(\frac{2 \pi \mathrm{i} k}{n}\right), s_{i}=\sin \left(\frac{2 \pi \mathrm{i} k}{n}\right)$ with $k=f_{\text {het }} / f_{\text {ph }}$. While our benchtop prototype uses a commercial A/D converter card and software such as gcc and $f f t w$ in the PC for this preprocessing stage, the EM and also the flight model (FM) will perform this step in dedicated hardware (FPGA), since this stage achieves a data reduction by a factor of about 100 and hence greatly reduces the burden on the data management unit (DMU) which does the final processing among other tasks. Furthermore, the critical A/D converter timing can also be easily handled by the FPGA. Prototype phasemeters using this FPGA preprocessing stage are under construction in Birmingham and Hannover.

The second step combines these intermediate results with the final output by a moderate amount of floating-point operations in the DMU. The longitudinal signals $x_{1}, x_{1}-x_{2}$, etc are obtained from the total complex amplitude on one quadrant diode: $\mathrm{F}_{\Sigma}^{(1)}=\mathrm{F}_{A}+\mathrm{F}_{B}+$ $\mathrm{F}_{C}+\mathrm{F}_{D}$ on the first quadrant diode, and $\mathrm{F}_{\Sigma}^{(2)}$ for the second (reference) quadrant diode equivalently. The result ${ }^{8}$ is: $\varphi_{\text {long }}=\arg \left(\mathrm{F}_{\Sigma}^{(1)}\right)-\arg \left(\mathrm{F}_{\Sigma}^{(2)}\right)+n 2 \pi$, (with an integer $n$ from a phasetracking algorithm), and $\delta x=\delta \varphi_{\text {long }} \cdot \frac{\lambda}{2 \pi} \cdot 2 \cos (\alpha)$, where the constant factor $2 \cos (\alpha)$ results from the reflection at the test mass at slightly non-normal incidence (see [1]).

The phase noise in one phase measurement channel caused by the digitization process is given by

$$
\widetilde{\delta \varphi}=\frac{2^{-(b-1)}}{c \sqrt{3 f_{\text {samp }}}},
$$

where $b$ represents the effective number of bits of the A/D converter and $c \lesssim 1$ the interferometric contrast. For typical values of $b=14, c=0.8$ and $f_{\text {samp }}=50 \mathrm{kHz}$ a noise floor of $\widetilde{\delta \varphi} \approx 4 \times 10^{-7} \mathrm{rad} \mathrm{Hz}^{-1 / 2}$ results, which is well below the requirement. When a differential phase between two channels is measured, this number increases by $\sqrt{2}$.

Two types of alignment signals are computed independently on each diode: $\mathrm{F}_{\mathrm{Left}}=$ $\mathrm{F}_{A}+\mathrm{F}_{D}$ : amplitude in left half, $\mathrm{DC}_{\mathrm{Left}}=\mathrm{DC}_{A}+\mathrm{DC}_{D}$ : average in left half, $\mathrm{F}_{\mathrm{Right}}, \mathrm{F}_{\mathrm{Upper}}$, $\mathrm{F}_{\text {Lower }}, \mathrm{DC}_{\text {Right }}, \mathrm{DC}_{\text {Upper }}, \mathrm{DC}_{\text {Lower }}$ equivalently.

The DC (ratiometric) signals correspond to the 'usual' way of using a quadrant diode and measure the average lateral displacement of the two beams with respect to the centre of the quadrant diode:

$$
\Delta x=\frac{\mathrm{DC}_{\text {Left }}-\mathrm{DC}_{\text {Right }}}{\mathrm{DC}_{\Sigma}}, \quad \Delta y=\frac{\mathrm{DC}_{\text {Upper }}-\mathrm{DC}_{\text {Lower }}}{\mathrm{DC}_{\Sigma}} .
$$

The calibration factor from test mass tilt angle $\alpha$ to $\Delta x$ is (with several idealizations) given by $\mathrm{d}(\Delta x) / \mathrm{d} \alpha=2 \sqrt{2 / \pi} L / w$, where $L \approx 25-50 \mathrm{~cm}$ is the lever arm from test mass to photodiode, and $w \approx 0.5-1 \mathrm{~mm}$ the beam radius at the photodiode.

8 This method assumes that the heterodyne frequency $f_{\text {het }}$ is an integer multiple of $f_{\text {ph. }}$ In LTP both frequencies will be derived from a common master clock by digital division by appropriate integers and the effects of any Doppler shifts are expected to be negligible. In the presence of non-negligible Doppler shifts, either a time-domain window function or post-measurement correction formulae (in the DMU) can be applied. 

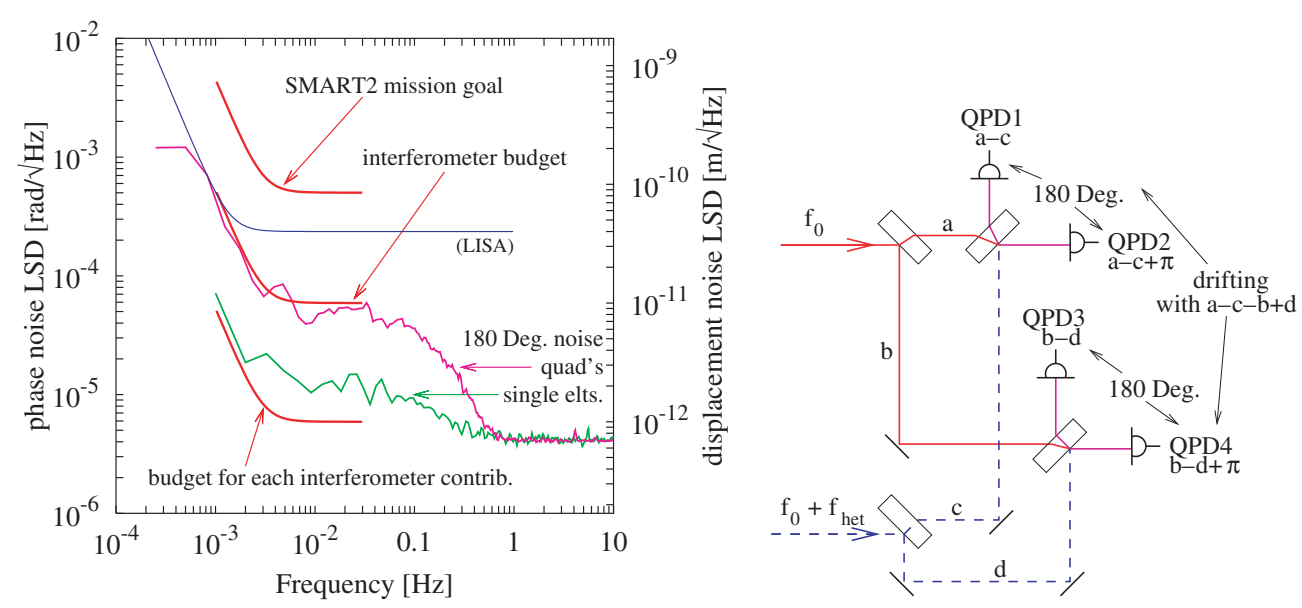

Figure 4. Noise level of the prototype interferometer. The right-hand diagram shows a sketch of the laboratory setup where $a, b, c$ and $d$ represent the pathlength-induced optical phase delays $2 \pi L / \lambda$, with constants due to optical components removed for convenience. The expressions $a-c$ etc indicate which phase is measured by the respective quadrant photodiode QPD.

The DWS signals, on the other hand, measure the angle between the two interfering wavefronts and thus provide complementary information:

$$
\Phi_{x}=\arg \left(\frac{\mathrm{F}_{\text {Left }}}{\mathrm{F}_{\text {Right }}}\right), \quad \Phi_{y}=\arg \left(\frac{\mathrm{F}_{\text {Upper }}}{\mathrm{F}_{\text {Lower }}}\right) .
$$

Their calibration factor (again with several idealizations) is $\mathrm{d}\left(\Phi_{x}\right) / \mathrm{d} \alpha=4 \sqrt{2 \pi} w / \lambda$, where $\lambda=1064 \mathrm{~nm}$ is the laser wavelength.

In detail, both calibration factors depend on parameters such as the beam shape, photodiode geometry, beam power ratios etc, so that a calibration by measurement is necessary (as opposed to the longitudinal signals above). Both the longitudinal and the alignment signals will be used as error signals in feedback loops that stabilize the test masses in some planned modes of operation.

The phasemeter will exist in two identical copies, each processing 16 channels arranged so that even if one phasemeter fails completely, all information is still available. The power consumption of the phasemeter depends mainly on the sampling rate $f_{\text {samp }}$ and it is expected that the preliminary power budget of $15 \mathrm{~W}$ can be met.

\section{Prototype experiment}

A table-top laboratory prototype of the interferometer was built and characterized in Hannover. It consists of two similar interferometers (instead of four), whose phases are compared. They are built on a standard optical bench and are functionally as close as possible to the planned flight hardware. Longitudinal, alignment and frequency fluctuation signals are in good agreement with theory. The main emphasis of our investigations has been the study of noise sources. The right-hand drawing in figure 4 shows a sketch of the two interferometers. While the phase noise measured between them (e.g., between QPD2 and QPD4) is relatively high due to the unstabilized environment, the two complementary outputs from one interferometer (e.g., QPD1 and QPD2) should have a constant $180^{\circ}$ phase shift. In reality, however, they show a variety of noise sources that can be studied. The left-hand side of figure 4 shows the 
results of these $180^{\circ}$ measurements with both single-element and quadrant diodes. Among the noise sources that have been studied are the following:

Non-simultaneous sampling. This has been fixed with a bank of S-H amplifiers; the new FPGA phasemeter will have inherent simultaneous sampling.

Electrical interference. This has an effect, but is difficult to reproduce. The new phasemeter will have optical coupling to the PC.

Thermal drift in steep antialiasing filter. This is now limiting at $f<10 \mathrm{mHz}$, and will be fixed in the new phasemeter with higher $f_{\text {samp }}$ and lower steepness requirements.

Beam jitter (pointing noise) with too small apertures or parasitical beamsplitter reflections. This is the dominating noise source between 30 and $300 \mathrm{mHz}$. On the photodiode there is a beam parameter mismatch between the two interfering beams due to asymmetry in the fibre output couplers and different pathlengths. The phase of the interference pattern hence has, e.g., a positive area in the centre and a negative ring around it. Beam jitter in conjunction with apertures or the insensitive slit of the quadrant photodiode then causes variable parts of that inhomogeneous phase pattern to be cut and hence a variation in the integrated phase. This phenomenon is still under investigation. In the very quiet environment of LTP, it is expected to be much smaller than on our bench (where the noise level already fulfils the specifications).

In fact, these $180^{\circ}$ measurements share most noise sources that will also appear in the real interferometer (apart from real pathlength fluctuations) and are hence an excellent study tool towards a complete understanding of the noise behaviour.

\section{Conclusion}

Detailed laboratory investigations have demonstrated the feasibility of the interferometer concept, the alignment measurement techniques and the phasemeter operation. Several noise sources have been identified and removed. An engineering model of the optical bench is nearly complete and will undergo extensive tests in the near future. While the requirements for the LISA phasemeter are different due to large Doppler shifts and higher frequencies, the experience obtained with the LTP phasemeter will be valuable in designing a phasemeter for LISA.

\section{Acknowledgments}

The authors acknowledge support from DLR project 50OQ0002, from ESA/ESTEC 'Optical bench' contract (and useful contributions from its technical officer M te Plate).

\section{References}

[1] Astrium et al 2000 LISA: study of the laser interferometer space antenna Final Technical Report, ESTEC Contract No 13631/99/NL/MS, Report No LI-RP-DS-009

[2] Spero R et al 2004 Class. Quantum Grav. 21 S589

[3] Heinzel G et al 2003 Interferometry for the LISA technology package (LTP) aboard SMART-2 Class. Quantum Grav. 20 S153-61 